\title{
Insight into Two ABC Transporter Families Involved in Lantibiotic Resistance
}

\author{
Rebecca Clemens, Julia Zaschke-Kriesche, Sakshi Khosa and Sander H. J. Smits*
}

Institute of Biochemistry, Heinrich-Heine-University Duesseldorf, Duesseldorf, Germany

Antimicrobial peptides, which contain (methyl)-lanthionine-rings are called lantibiotics. They are produced by several Gram-positive bacteria and are mainly active against these bacteria. Although these are highly potent antimicrobials, some human pathogenic bacteria express specific $\mathrm{ABC}$ transporters that confer resistance and counteract their antimicrobial activity. Two distinct $\mathrm{ABC}$ transporter families are known to be involved in this process. These are the Cpr- and Bce-type ABC transporter families, named after their involvement in cationic peptide resistance in Clostridium difficile, and bacitracin efflux in Bacillus subtilis, respectively. Both resistance systems differentiate to each other in terms of the proteins involved. Here, we summarize the current knowledge and describe

OPEN ACCESS

Edited by:

Richard J. Bingham, Department of Biological Sciences, University of Huddersfield, United Kingdom

Reviewed by: Anastassios C. Papageorgiou, University of Turku, Finland Christopher Cooper, University of Huddersfield, United Kingdom

*Correspondence: Sander H. J. Smits sander.smits@hhu.de

Specialty section This article was submitted to Structural Biology,

a section of the journal

Frontiers in Molecular Biosciences

Received: 28 August 2017 Accepted: 08 December 2017 Published: 22 January 2018

Citation:

Clemens R, Zaschke-Kriesche J, Khosa S and Smits SHJ (2018) Insight into Two ABC Transporter Families Involved in Lantibiotic Resistance.

Front. Mol. Biosci. 4:91. doi: 10.3389/fmolb.2017.00091 the divergence as well as the common features present in both the systems to confer lantibiotic resistance.

Keywords: lanthionine ring, lantibiotic, nisin, resistance, antimicrobial peptide, L. lactis

\section{INTRODUCTION}

The urging need for novel antibiotics has put small antimicrobial peptides (AMPs) into a particular focus. Especially, a large group of peptides called bacteriocins have been extensively studied for an application purpose as novel antibiotics. Bacteriocins are small, ribosomally-synthesized peptides of which some display a high potent antimicrobial activity (Tagg et al., 1976; Cotter et al., 2005b) and have been already used since decades as food preservatives or as antibiotic alternatives in biomedical applications (Cleveland et al., 2001; Cotter et al., 2012).

A large group within the bacteriocin family, are lanthionine containing antibiotics termed lantibiotics. These lantibiotics are post-translationally modified peptides that contain dehydrated amino acids (Dehydrobutyrine and/or Dehydroalanine) and other unusual amino acid modifications (Jung, 1991; Willey and van der Donk, 2007; Bierbaum and Sahl, 2009; Alvarez-Sieiro et al., 2016). The Michael addition of a neighboring cysteine side chain residue to these dehydrated amino acids results in the formation of characteristic thioether bridges called lanthionine rings. These rings are primarily crucial for their high antimicrobial activity against mainly Gram-positive bacteria. The well-known lantibiotics nisin, gallidermin, and subtilin are highlighted in Figure 1. Lantibiotics are highly potent and nanomolar concentrations are already enough to fulfill their antimicrobial activity as observed for example for nisin produced by Lactococcus lactis species or subtilin produced by Bacillus subtilis (Delves-Broughton et al., 1996; Chatterjee et al., 2005).

In comparison to their high antimicrobial activity against Gram-positive bacteria, lantibiotics display a reduced effectiveness against Gram-negative bacteria. Many lantibiotics bind to lipid II or other peptidoglycan precursor inducing inhibition of cell wall synthesis. Some lantibiotics can subsequently form pores which lead to membrane leakage and rapid cell death (Héchard and Sahl, 2002; Bierbaum and Sahl, 2009). Due to their nanomolar activity, in combination with high stability 

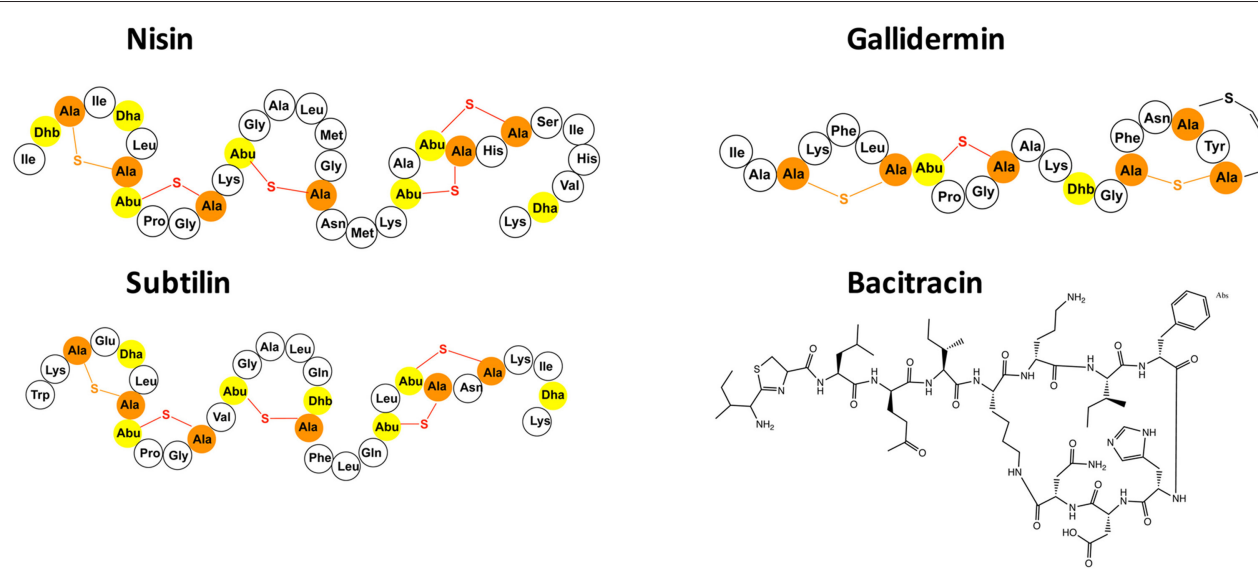

FIGURE 1 | Selected presentation of the lantibiotics nisin, gallidermin and subtilin as well as the bacteriocin bacitracin. The dehydrated amino acids and the cysteines of the lantibiotics are highlighted in yellow and orange. The (methyl-)lanthionine rings are visualized in orange and red.

against proteolytic digestion, lantibiotics are considered potential compounds for novel medical treatment.

A well-studied member of lantibiotics is nisin, which is produced by some L. lactis strains. It was shown that it is effective against the treatment of bacterial mastitis, methicillin-resistant Staphylococcus aureus (MRSA) and enterococcal infections (Brumfitt et al., 2002). Gallidermin and epidermin, produced by Staphylococcus gallinarum and Staphylococcus epidermidis, respectively, are some other examples of lantibiotics (Cotter et al., 2005a) and are associated with the treatment of acne, eczema, folliculitis, and impetigo.

The lantibiotic producer strains with a few exceptions, usually contain a single gene cluster, on which the structural genes for the lantibiotic itself, as well as for the modification and transport across the cellular membrane are located (Chatterjee et al., 2005; Willey and van der Donk, 2007; Alkhatib et al., 2012; Singh and Sareen, 2014). In many gene clusters, these genes are upregulated via a distinct two-component system (TCS) consisting of a histidine kinase $(\mathrm{HK})$ and a response regulator (RR), which are located on the same gene cluster (Qiao et al., 1996). The upregulation is auto induced by its own lantibiotic (Kuipers et al., 1995).

Due to the high convergence of these gene clusters, it has been possible to detect potential lantibiotic gene clusters within newly sequenced genomes using in silico techniques (van Heel et al., 2013a). Such genome mining approaches have identified novel lantibiotic gene clusters in several species, such as the genes encoding for maddinglicin from Clostridium maddingley, agalacticin from Streptococcus agalactiae, bagelicin from Streptococcus suis and moraviensicin from Enterococcus moraviensis (van Heel et al., 2013b; Tracanna et al., 2017). These novel and mostly exotic lantibiotics can be expressed, modified and secreted by L. lactis using the well-characterized nisin biosynthetic machinery (van Heel et al., 2013b).

In order to prevent the activity of the secreted lantibiotic against their own membrane, the lantibiotic gene cluster contains additional genes (lanI and lanFEG), which form a lantibiotic (auto-)immunity system (Alkhatib et al., 2012). The lanI and lanFEG genes are conserved to a certain extent throughout the lantibiotic expressing bacteria (Alkhatib et al., 2012). Here LanI is a membrane-associated lipoprotein, which binds to the lantibiotic and thereby lowers the concentration of the lantibiotic reaching the membrane. Additionally, LanFEG forms an ABC transporter localized in the cellular membrane which effluxes the lantibiotic prior to pore formation (Stein et al., 2003, 2005; Draper et al., 2008, 2015).

Despite the odds, resistance against lantibiotics does exist and different resistance mechanisms have been unraveled so far. Resistance mechanisms comprise of modification in peptidoglycan or the cellular membrane (e.g., changes in phospholipid or fatty acid composition) as well as cell membrane modifications, such as lipopolysaccharides which are attached to the outer layer of the outer membrane of Gram-negative bacteria (Draper et al., 2015). Furthermore, some TCSs could be linked to lantibiotic resistance by upregulating the transcription of resistance-associated genes upon the presence of lantibiotic within the habitat of the bacteria. Other mechanisms resulting in resistance are the assembly of biofilms or the expression of resistance proteins such as the nisin resistance protein (NSR) found to be upregulated in nisin non-producing strains, inactivating nisin by specific proteolytic degradation (Sun et al., 2009). The lantibiotic resistance mechanisms have been nicely reviewed in detail in Draper et al. (2015).

Recently, several gene clusters were identified in various human pathogenic bacteria, which encode a lantibiotic resistance system based on the overexpression of membrane embedded proteins, that includes the presence of an $\mathrm{ABC}$ transporter (Khosa et al., 2013).

The expression of proteins within these gene clusters result in a detectable lantibiotic resistance. For example, resistance against nukacin ISK-I and lacticin 481 in Streptococcus mutans is mediated by the expression of lcrSR-lctFEG genes (KawadaMatsuo et al., 2013a), while the expression of $\operatorname{cpr} A B C K-R$ operon in Clostridium difficile results in resistance against 
different lantibiotics. Here, nisin, mutacin 1140, subtilin, and gallidermin were tested and resistance was observed (McBride and Sonenshein, 2011; Suárez et al., 2013). Furthermore, the proteins located on the $n s r$ operon from $S$. agalactiae are together conferring resistance against nisin $\mathrm{A}$, nisin $\mathrm{H}$, and gallidermin (Khosa et al., 2016a,b; Reiners et al., 2017). All these resistance operons are characterized by the presence of a TCS consisting of a HK and a RR; as well as a membrane-embedded ATP-binding cassette (ABC) transporter (Gebhard, 2012; Khosa et al., 2013; Suárez et al., 2013). In some cases, an extra gene encoding a membrane-associated lipoprotein or a specific serine protease is present.

Upon examining these operons in detail, it was observed that the $\mathrm{ABC}$ transporters are responsible for lantibiotic resistance and can be divided into two groups: the CprABC-type and the BceAB-type $\mathrm{ABC}$ transporter family, both conferring resistance against lantibiotics and/or antimicrobial peptides in general.

Within this review, we will highlight these two lantibiotic resistance $\mathrm{ABC}$ transporter families and their corresponding gen clusters.

\section{GENE CLUSTER ORGANIZATION OF ABC TRANSPORTERS INVOLVED IN LANTIBIOTIC RESISTANCE}

The common feature of both operon types is the presence of a TCS, which upregulates the other genes by an external stimulus via binding of the lantibiotic. Furthermore, they both consist of an $\mathrm{ABC}$ transporter, which is thought to expel the lantibiotic once it has reached the bacterial membrane (Figure 2). In general, $\mathrm{ABC}$ transporter comprises of a transmembrane domain (TMD) and a nucleotide-binding domain (NBD). The NBD dimerizes upon binding of ATP, which is subsequently hydrolysed and the energy released is used to induce a conformational change within the TMD allowing substrate export or import. Furthermore, in some of these operons there is also the presence of either a lipoprotein or a membrane-associated specific protease.

\section{CprABC-TYPE RESISTANCE OPERONS}

Lantibiotic resistance operons belonging to the Cpr group contain three different genes encoding for an $\mathrm{ABC}$ transporter (one for the NBD and two different TMDs; highlighted in blue, Figure 2) and genes encoding a $H K$ and $R R$, which build up the TCS. These transporters belong to the ABC-type 2 sub-family and on a genetic level closely resemble the immunity systems found in lantibiotic producing strains. This group is named after the most prominent member, the CprABC transporter from C. difficile, which confers resistance against nisin and gallidermin (McBride and Sonenshein, 2011; Suárez et al., 2013). Here, the $\mathrm{NBD}$ is encoded by $c \operatorname{pr} A$ while $c \operatorname{pr} B$ and $\operatorname{cprC}$ encode the two TMDs. CprB and CprC are of similar size and are predicted to contain six transmembrane helices each. Both CprB and CprC form a functional transporter together in the membrane as a heterodimer (Figure 3).
Other members of this group are lcrSR-lctFEG and $n s r F E_{1} E_{2} G-X R K$, both present in the genome of $S$. mutans (Figure 2). The encoded TMDs of this group contain six predicted transmembrane helices. They are upregulated via one promoter suggesting that they are expressed in equal stoichiometry. These $\mathrm{ABC}$ transporters, are closely related to the immunity $\mathrm{ABC}$ transporter LanFEG family, which consists of the NBD LanF and two TMDs LanE and LanG (as an example, the nisin immunity system called NisFEG is highlighted in Figure 2). These LanFEG ABC transporters are co-expressed in lantibiotic producer strains and have been shown to expel lantibiotics from the membrane preventing a suicidal effect (Stein et al., 2003, 2005; Alkhatib et al., 2012; AlKhatib et al., 2014b). The LanFEG genes are, in contrast to the Cpr group, encoded in a larger operon which also include genes for biosynthesis and transport machinery of the produced lantibiotic. Due to the operon similarity, one can assume that the cpr operons are evolutionary linked to the producing strains. Here however, only the genes for the resistance proteins are present and none of the biosynthetic machinery. Next to the ABC transporter genes, a TCS is present which consists of the HK and RR, which are distantly located on the chromosome (Suárez et al., 2013) (highlighted in green, Figure 2). These TCSs transfer the stimuli provided by the externally present lantibiotic into the cell and induce transcription of the genes.

\section{BceAB RESISTANCE OPERON}

Lantibiotic resistance operons belonging to the Bce group contain genes encoding for an $\mathrm{ABC}$ transporter, i.e., two different genes encoding one NBD and one large TMD (highlighted in blue, Figure 2). Additionally, a TCS is present consisting of a histidine kinase and response regulator. BceAB-type (ABC) transporters are putatively involved in antimicrobial peptide as well as lantibiotic removal from the lipid membrane (Gebhard and Mascher, 2011). They have been named after the transporter system from $B$. subtilis, which till date is the best characterized representative of $\underline{B}$ acitracin $\underline{\boldsymbol{e}} \underline{\mathrm{ffl}} \mathrm{ux}$ (Bce) transporters, conferring resistance against the antimicrobial peptide bacitracin (Ohki et al., 2003; Rietkötter et al., 2008).

Based on the Transport Classification Database (TCDB), BceAB-type transporters belong to the peptide 7 exporter family (Saier et al., 2009). The BceAB-type transporters are composed of two components, a NBD (BceA) and a single TMD (BceB) (Figure 2). The TMD consists of ten predicted transmembrane helices (TMHs) and contain a large, extracellular domain $\left(\mathrm{ECD}_{L}\right.$ where $\mathrm{L}$ stands for lantibiotic) between transmembrane helices VII and VIII (Figure 3). This extracellular domain appears to be the hallmark of BceAB-type transporters and consists of 200-250 amino acids (Ohki et al., 2003; Rietkötter et al., 2008; Khosa et al., 2013; Figure 3).

Bioinformatically, $\mathrm{ECD}_{L}$ are easy to detect and have been for example identified in the TMDs of the bacitracin resistanceassociated $\mathrm{ABC}$ transporter $\mathrm{Bce} \mathrm{AB}$ in Bacillus species (Rietkötter et al., 2008), in the bacitracin and nisin resistance-associated ABC transporter VraDE in S. aureus (Hiron et al., 2011) and the nisin 


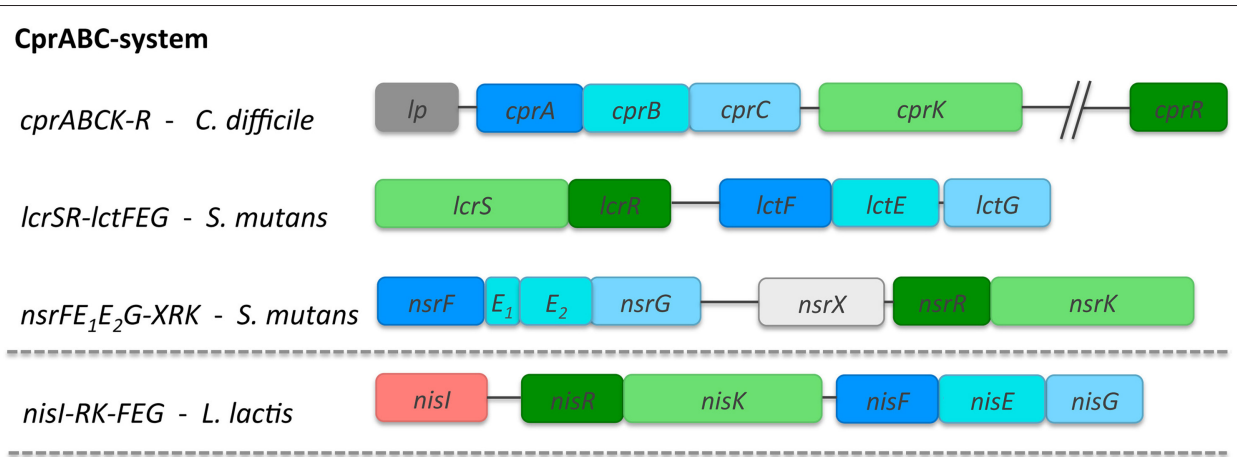

\section{BceAB system}

bceRS-AB - B. subtilis

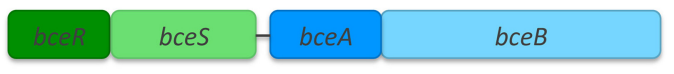

braSR-vraDE - S. aureus

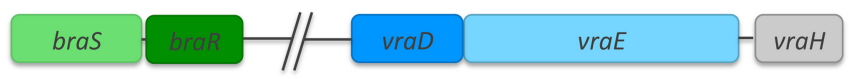

nsrFP-RK - S. agalactiae

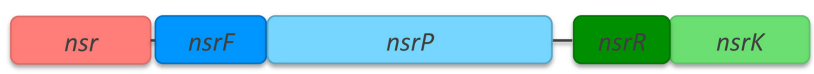

FIGURE 2 | Overview of the lantibiotic resistance operons belonging to the Cpr- and Bce- type systems. Three operon structures, each are highlighted as representatives for the Cpr and Bce group. For Cpr-type these are cprABCK-R from C. difficile, nsrFE $E_{2} G-X R K$ and IcrSR-IctFEG from S. mutans. Additionally, the nisRK-FEG system from $L$. lactis involved in nisin immunity is also highlighted. For Bce-type, the three representatives comprise of bceRS-AB from $B$. subtilis, braSR-vraDE from S. aureus and the nsrFP-RK system from S. agalactiae. The size of the genes corresponds directly with the gene length as deposited in the NCBI database. The TCSs with RR (dark green) and HK (light green); and the ABC transporters are shown in different shades of blue. In the bceAB system, the NBD is dark blue while the TMD is shown in light blue. The additional TMD present in the cprABC systems is shown in cyan. The proteins, which are part of the operon but the function has not been determined so far are shown in gray. In case of the nis and nsr operons, an additional membrane-associated protein is present which is colored in red.

resistance-associated NsrFP in S. agalactiae (Khosa et al., 2013). These $\mathrm{ECD}_{L}$ are found to be crucial for resistance determinants as they are supposed to recognize the lantibiotic extracellularly and subsequently induce the TCS-dependent signal transduction within the cell (Rietkötter et al., 2008; Hiron et al., 2011). Initial substrate binding usually occurs via these $\mathrm{ECD}_{L}$, however, experimental evidence for this only has been indirectly proven via knockout studies (Falord et al., 2012).

In the Bce group, a BceRS-type TCS has co-evolved (Heijenoort, 1994; Dintner et al., 2011) composed of a response regulator (BceR) and a histidine kinase (BceS). The latter consists of two transmembrane helices with a short extracellular located loop of $\sim 25$ amino acids. Such a small loop is unusual for HKs, which normally consists of roughly 115-125 amino acids. This suggested that the TCS lacks an extracellular domain normally present to detect an external stimulus and is therefore, categorized as a member of the intramembrane-sensing histidine kinase family (Mascher et al., 2003; Mascher, 2006). Members of this family have been shown to be responsible for the upregulation of the corresponding $\mathrm{ABC}$-transporter in the presence of its specific lantibiotic (Staron et al., 2011).

In summary, three CprABC-type as well as BceAB-type $\mathrm{ABC}$ transporters are mentioned in detail, which are all well studied so far in order to highlight both their functional properties and the differences between these two groups. Within the CprABC group, we have included the NisFEG
ABC transporter, which confers immunity against nisin in the producer strains. General characteristics including protein sequence, size, and function of these systems are listed in Tables 1, 2.

\section{CprABC RESISTANCE SYSTEMS The CprABCK-R System from C. difficile}

The operon of the $\operatorname{cpr}$ (cationic antimicrobial peptide resistance) system from $C$. difficile consists of an $\mathrm{ABC}^{-}$transporter and a TCS. The genetic organization of the Cpr system resembles the immunity system found in lantibiotic producing strains (see above).

The CprABC transporter from $C$. difficile is encoded by three different proteins: a nucleotide binding domain called CprA (26 $\mathrm{kDa})$ and the two transmembrane domains called CprB and CprC (27 and $29 \mathrm{kDa}$, respectively; Figure 3 and Table 1). Both of the TMDs have six predicted transmembrane helices and form a functional heterodimer (Table $\mathbf{1}$ ).

The TCS is composed of a RR cprR (CD3320) and a HK cprK (CD1352). The histidine kinase of the Cpr system contains an extracellular loop (113 aa) (Table 1), which has been proposed to be involved in sensing. Such a loop is a general feature of histidine kinases. The regulator does not directly belong to the cpr operon and is distantly located on the chromosome (McBride and Sonenshein, 2011; Suárez et al., 2013). 


\section{A Mode of action}

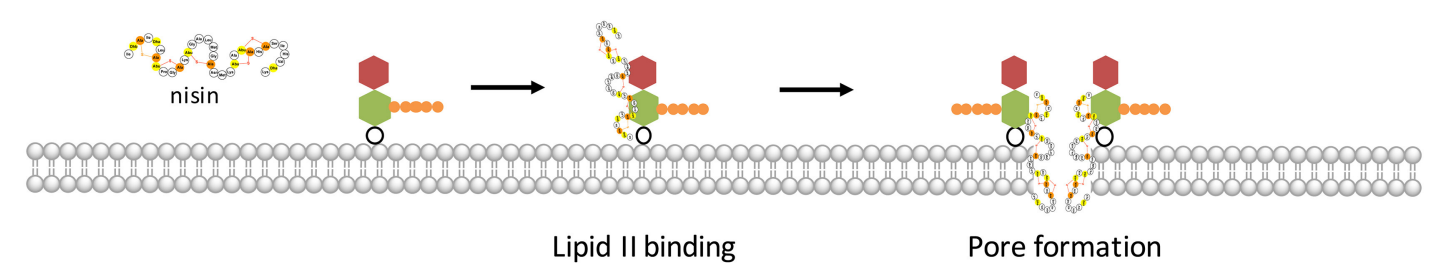

B CprABC type
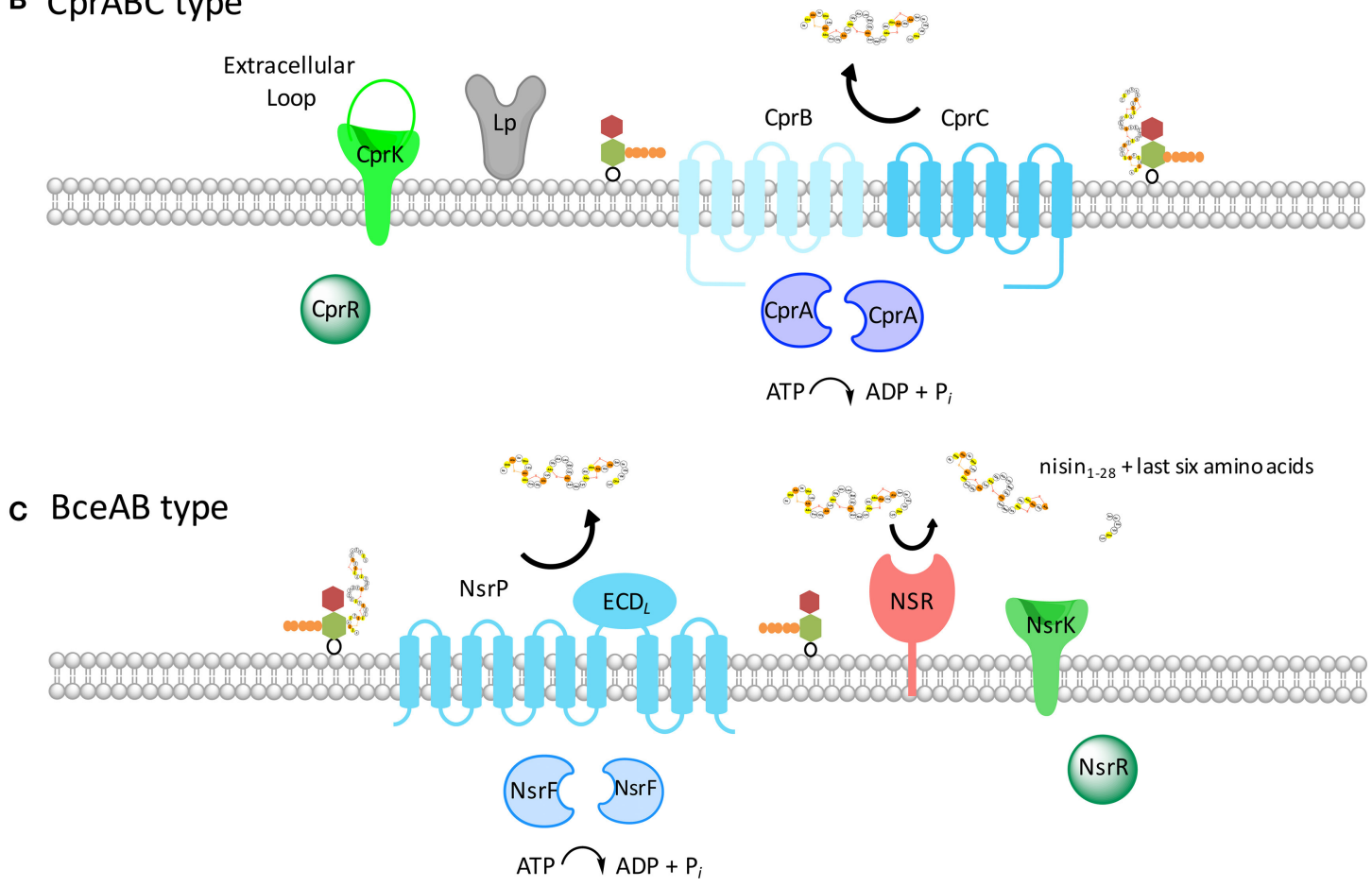

FIGURE 3 | Schematic representation of the two resistance systems of $C$. difficile and S. agalactiae. (A) The lantibiotic nisin A (see Figure 1) binds to the cell wall precursor lipid II, depicted with the $\mathrm{N}$-acetyl glucosamine colored in red, the $\mathrm{N}$-acetyl muramic acid shown in green and the pentapeptide presented in orange. The binding via the first two lanthionine rings of nisin to lipid II results growth inhibition and subsequently in pore formation. This results in rapid cell death already at nanomolar nisin concentrations. (B) The proteins encoded by the cpr operon are shown with the ABC transporter CprABC (depicted in blue) and the two-component system CprRK (depicted in green). The extracellular loop of the histidine kinase has also been shown. The lipoprotein adjacent to CprABC is depicted in gray. (C) The Nsr system of $S$. agalactiae is highlighted. The two-component system NsrRK and an efflux ABC transporter NsrFP are depicted in green and blue, respectively. The $E C D_{L}$ present in the $A B C$ transporter in between transmembrane helices seven and eight is indicated in cyan. Furthermore, an extra membrane-associated serine protease SaNSR present in this system is shown in red. SaNSR is a serine protease, which cleaves the last six amino acids of nisin off.

Within C. difficile, the cpr system has been shown to confer resistance against several lantibiotics such as nisin $\mathrm{A}$, gallidermin, and subtilin. Although these lantibiotics are quite different in their amino acid composition, however the first two lanthionine rings are structurally conserved in their tertiary structure (Suárez et al., 2013), suggesting the importance of this region for the cpr genes to recognize lantibiotics. It was proposed that the lanthionine ring along with the proline and glycine residues form the sequence motif recognized by CprK resulting in signaling of the TCS CprK-CprR (Suárez et al., 2013).

The genes encoding the $\mathrm{ABC}$ transporter $c p r A B C$ are regulated by $c p r K-c p r R$ and are found adjacent to $c p r K$ in the genome. Insertional disruption of one of the transporter genes resulted in significant decrease in resistance against both nisin A and gallidermin. Hence, this TCS and ABC transporter pair contributes to the resistance of $C$. difficile toward many lantibiotics (Suárez et al., 2013). It has been shown that the addition of nisin induced the expression of CprABC, so it could be proven that the CprR is responsible for the upregulation (McBride and Sonenshein, 2011; Suárez et al., 2013).

Additionally, adjacent of the cprABC gene cluster, a lipoprotein is present (CD1348). Although, no involvement in lantibiotic resistance has been described so far, the genetic context resembles the BceAB system found in $S$. agalactiae, which contains the SaNSR protein, a membrane associated resistance protein (see below). Interestingly, the lipoprotein is not upregulated by the presence of a lantibiotic or antimicrobial peptide and displays a basal expression level (Suárez et al., 2013). 
TABLE 1 | Comparison of the different Cpr-type resistance systems with the TCS and the $\mathrm{ABC}$ transporter.

\begin{tabular}{|c|c|c|c|c|}
\hline & CprABCK-R & LcrSR-LctFEG & $N_{s r F E} E_{2} G-X R K$ & NisRK-FEG \\
\hline Organism & $\begin{array}{l}\text { C. difficile } \\
\text { (Strain630) }\end{array}$ & $\begin{array}{l}\text { S. mutans } \\
\text { (UA159) }\end{array}$ & $\begin{array}{l}\text { S. mutans } \\
\text { (UA159) }\end{array}$ & $\begin{array}{l}\text { L. lactis } \\
\text { (NZ9700) }\end{array}$ \\
\hline $\begin{array}{l}\text { Defense } \\
\text { category }\end{array}$ & Resistance & Resistance & Resistance & Immunity \\
\hline $\begin{array}{l}\text { Resistance } \\
\text { against }\end{array}$ & $\begin{array}{l}\text { nisin A, subtilin, } \\
\text { gallidermin, } \\
\text { mutacin } 1140 \text {, } \\
\text { cinnamycin }\end{array}$ & $\begin{array}{l}\text { lacticin 481, } \\
\text { nukacin ISK-I }\end{array}$ & nisin $A$ & nisin $A$ \\
\hline TMD [aa] & $\begin{array}{l}238(B) \\
252(C)\end{array}$ & $\begin{array}{l}246(E) \\
242(G)\end{array}$ & $\begin{array}{l}82 \text { (E1) } \\
171 \text { (E2) } \\
248(\mathrm{G})\end{array}$ & $\begin{array}{l}242(E) \\
214(G)\end{array}$ \\
\hline $\begin{array}{l}\text { Oligomeric } \\
\text { state }\end{array}$ & $\begin{array}{l}\text { Dimer } \\
(B+C)\end{array}$ & $\begin{array}{l}\text { Dimer } \\
(E+G)\end{array}$ & $\begin{array}{l}\text { Trimer } \\
(\mathrm{G}+\mathrm{E} 1+\mathrm{E} 2)\end{array}$ & $\begin{array}{l}\text { Dimer } \\
(E+G)\end{array}$ \\
\hline TMHs & $6+6$ & $6+6$ & $6+2+4$ & $6+6$ \\
\hline NBD [aa] & 235 & 255 & 234 & 225 \\
\hline $\begin{array}{l}\text { Response } \\
\text { regulator [aa] }\end{array}$ & 219 & 229 & 219 & 229 \\
\hline $\begin{array}{l}\text { Histidine } \\
\text { kinase [aa] }\end{array}$ & 462 & 437 & 460 & 447 \\
\hline $\begin{array}{l}\text { Loop-cont. } \\
\text { protein }\end{array}$ & Kinase & Kinase & Kinase & Kinase \\
\hline Loop size [aa] & 113 & 117 & 116 & 112 \\
\hline
\end{tabular}

TABLE 2 | Comparison of the different Bce-type resistance machineries with the TCS and the ABC transporter.

\begin{tabular}{llll}
\hline & Bce $\boldsymbol{R S}$ - $\boldsymbol{A B}$ & BraSR-VraDE & NsrFP- $\boldsymbol{R} \boldsymbol{K}$ \\
\hline Organism & $\begin{array}{l}\text { B. subtilis } \\
\text { (Strain 168) }\end{array}$ & $\begin{array}{l}\text { S. aureus } \\
\text { (USA300) }\end{array}$ & $\begin{array}{l}\text { S. agalactiae } \\
\text { (COH1) }\end{array}$ \\
Defense category & Resistance & Resistance & Resistance \\
Resistance against & bacitracin, & bacitracin, & nisin A, \\
& actagardine, & nisin A, & nisin H, \\
TMD & mersacidin & daptomycin & gallidermin \\
Oligomeric state & 646 & 626 & 651 \\
TMHs & Monomer & Monomer & Monomer \\
NBD [aa] & 10 & 10 & 10 \\
Response regulator [aa] & 231 & 252 & 250 \\
Histidine kinase [aa] & 334 & 221 & 222 \\
ECD cont. protein & TMD & 295 & 262 \\
ECD L size[aa] & 216 & TMD & TMD \\
\hline
\end{tabular}

\section{The LcrSR-LctFEG and NsrFE $\mathrm{E}_{2} \mathrm{G}-\mathrm{XRK}$ Resistance Systems}

S. mutans (UA159) has two resistance systems, namely, the LcrSR-LctFEG and $\mathrm{NsrFE}_{1} \mathrm{E}_{2} \mathrm{G}-\mathrm{XRK}$. Both consist of a TCS and an $\mathrm{ABC}$ transporter.

The LcrSR-LctFEG system confers resistance against lacticin 481 and nukacin ISK-I as determined with growth inhibition analyses (Kawada-Matsuo et al., 2013a,b).

Within this system, LcrR $(26 \mathrm{kDa})$ is the RR and the LcrS $(50$ $\mathrm{kDa}$ ) is the $\mathrm{HK}$, which also contains an extracellular sensing loop (117aa) (Table 1). The ABC transporter consists of three different domains. The NBD LctF (29 kDa), which is important for the ATP binding and hydrolysis, and two transmembrane domains, LctE $(28 \mathrm{kDa})$ and LctG $(27 \mathrm{kDa})$, which each consist of six transmembrane helices each (Kawada-Matsuo et al., 2013b).

The $\mathrm{NsrFE}_{1} \mathrm{E}_{2} \mathrm{G}-\mathrm{XRK}$ system contains a TCS NsrRK with the NsrR $(25 \mathrm{kDa})$ as RR and the NsrK $(53 \mathrm{kDa})$ as HK containing an extracellular loop of 116 amino acids. The ABC transporter system contains four proteins: the NBD NsrF $(26 \mathrm{kDa})$ and the three TMDs $\mathrm{NsrE}_{1} \mathrm{E}_{2} \mathrm{G}$. Here, the NsrG $(28 \mathrm{kDa})$ has six transmembrane helices, the $\mathrm{NsrE}_{1}(10 \mathrm{kDa})$ has two and $\mathrm{NsrE}_{2}$ $(20 \mathrm{kDa})$ has four transmembrane helices, so in total 12 , which is similar to the other known ABC transporters. However, for $\mathrm{NsrFE}_{1} \mathrm{E}_{2} \mathrm{G}$ resistance against only nisin $\mathrm{A}$ was observed, which was examined using deletional mutants within the NsrRK system. For other tested lantibiotics like nukacin ISK-1, no resistance could be observed (Kawada-Matsuo et al., 2013b).

\section{The NisFEG Immunity Transporter from L. lactis}

In the self-immunity system of nisin producing strains, the cytoplasmic NisF (25 kDa) is composed of 225 amino (Siegers and Entian, 1995). Additionally, NisE (28 kDa) and NisG $(24 \mathrm{kDa})$ are predominantly hydrophobic proteins, that form together an integral membrane part of the ABC transporter and are composed of six transmembrane helices each (Siegers and Entian, 1995). Using sequence similarity searches NisFEG likely exhibits a 2:1:1 stoichiometry to form a functional lantibiotic immunity LanFEG transporter (Siegers and Entian, 1995). Various gene knockout studies have shown that out of all the three genes of the $\mathrm{ABC}$ transporter, deletion of nisE gene has the most detrimental effect on immunity (Siegers and Entian, 1995).

The primarily function of NisFEG in providing immunity to the producer strain is the efflux of nisin molecules from the membrane before they can form pores (Stein et al., 2003; AlKhatib et al., 2014b). A similar function has been identified for the subtilin immunity ABC transporter SpaFEG, which is able to transport subtilin from the cytoplasmic membrane directly back into the exterior (Stein et al., 2005).

When expressed in the nisin sensitive L. lactis strain NZ9000, which does not carry the immunity genes nisI and nisFEG within its genome, NisFEG confers seven to eight fold of immunity when expressed alone (AlKhatib et al., 2014b).

The substrate specificity of NisFEG has been extensively studied. It has been shown that NisFEG recognizes the Cterminally located lanthionine ring and the last six amino acids of nisin as a reduction of $50 \%$ in the immunity provided by NisFEG was seen upon deletion of either of them (AlKhatib et al., 2014b).

\section{THE Bce RESISTANCE SYSTEMS}

\section{BceRS-AB System from $B$. subtilis}

The BceRS-AB system from $B$. subtilis consists of the $A B C$ transporter, with NBD BceA (28 kDa) and TMD BceB $(72 \mathrm{kDa})$, and the TCS with the response regulator $\mathrm{BceR}(27 \mathrm{kDa})$ and the histidine kinase BceS (39 kDa) (Figure 3 and Table 2). Various 
growth inhibition assays of strains expressing BceRS-AB and several deletion mutants have shown, that this system mediates resistance against actagardine, mersacidin, and bacitracin (Ohki et al., 2003; Staron et al., 2011).

For signal transduction purposes, both the BceAB ABC transporter as well as the TCS need to be present to confer a signal transduction inducing upregulation of the genes encoded on the operon. This is an unusual mode of signal transduction, as the HK cannot sense bacitracin alone, and needs the presence of the $A B C$ transporter to sense the substrate in the surrounding. Furthermore, an ATP hydrolysis deficient transporter mutant highlighted that hydrolysis is required for this signaling process (Rietkötter et al., 2008). Based on random mutagenesis studies it was shown that the C-terminal part of the TMD BceB, specifically up to helix VIII is important for signaling and resistance of the BceAB-RS system in B. subtilis (Kallenberg et al., 2013).

The BceAB from B. subtilis has been to the best of our knowledge, the only system which has been purified and shown to form a multicomponent complex with its designated TCS BceRS upon binding of bacitracin (Dintner et al., 2014). These biochemical analyses of the $\mathrm{Bce} A \mathrm{~B}$ and $\mathrm{BceRS}$ proteins showed that the TCS, more specifically the BceS module, and the transporter form a so-called sensory complex in the cytoplasmic membrane, where the kinase activity is relying on the $\mathrm{BceAB}$ transporter (Dintner et al., 2014). This further underlines the fact that the $\mathrm{BceAB}$ transporter from $B$. subtilis is directly involved in bacitracin sensing and consequently triggers the upregulation of its own gene by the TCS BceRS. This was further highlighted by mathematical modeling response dynamics of the Bce system, which suggested a direct correlation between the transport activity of $\mathrm{BceAB}$, and the BceS kinase signaling activity (Fritz et al., 2015).

BceAB-like transporters are thought to recognize the targetpeptide complex within the membrane and not the peptide as such (Bernard et al., 2007; Rietkötter et al., 2008). This idea is further strengthened by experiments suggesting that $\mathrm{BceAB}$ of B. subtilis does not export bacitracin, but instead acts as a flippase of the target molecule undecaprenyl pyrophosphate (UPP) to the cytoplasmic side of the membrane (Kingston et al., 2014), thereby removing the target of bacitracin. However, Surface Plasmon Resonance (SPR) spectroscopy studies of BceAB have shown that the TMD BceB binds bacitracin with a high affinity $\left(K_{D}\right.$ of $\left.60 \mathrm{nM}\right)$ in detergent solution and appears to be specific for the active bacitracin- $\mathrm{Zn}^{2+}$-complexed form (Dintner et al., 2014). Although these results do not rule out that a bacitracin-UPP complex is recognized by BceAB, it suggests an effluxing mechanism for bacitracin. Random mutagenesis studies further highlighted, that the C-terminal part of the TMD $\mathrm{BceB}$ up to helix VIII is important for the signaling and the resistance of the BceRS-AB system in B. subtilis (Kallenberg et al., 2013).

\section{The VraDE-BraRS from S. aureus}

The VraDE-BraRS system of $S$. aureus is a system composed of the NBD V V regulator BraR $(25 \mathrm{kDa})$, and the histidine kinase BraS $(34 \mathrm{kDa})$. This system has been identified in S. aureus since only two of the
16 TCSs present have been linked to the Bce family. Here, the TCS GraRS (Meehl et al., 2007) and the VraDE-BraRS system, mediate cationic antimicrobial peptide resistance (Hiron et al., 2011).

The VraDE-BraRS system of $S$. aureus confers resistance against bacitracin, nisin $\mathrm{A}$ and daptomycin as determined via growth inhibition experiments of $S$. aureus (Hiron et al., 2011; Popella et al., 2016). Transcriptional fusions using the operon promoter revealed increased expression when induced with increasing sub-lethal bacitracin and nisin concentrations as previously observed for the BceRS/BceAB module of $B$. subtilis (Ohki et al., 2003; Hiron et al., 2011). The TCS BraRS activates transcription of the BraDE and VraDE operons, encoding two $A B C$ transporters, which play distinct and original roles in bacitracin and nisin resistance. Here, $\mathrm{VraDE}$ is a specific detoxification system and is sufficient to confer resistance against bacitracin and nisin when expressed alone (Hiron et al., 2011) whereas BraDE and BraRS are involved in antibiotic sensing and signaling, respectively.

The $\mathrm{ABC}$ transporter VraDE is directly involved in bacitracin resistance. By using chimeric $\mathrm{ABC}$ transporter and domainswapping variants, where the extracellular loop of $\mathrm{VraD}$ was exchanged by the one of VraE, it was observed that the extracellular domain of $\mathrm{VraE}$ is the determinant for bacitracin specificity (Hiron et al., 2011; Popella et al., 2016). Furthermore, it was shown that VraH, a small transmembrane protein, is further an essential component of the VraDE complex to form the functional VraDEH complex. VraH of $S$. aureus JE2 is a positively charged C-terminus containing a conserved YYKRREEKGK motif. The cytoplasmic VraD interacts with the transmembrane protein VraH. This complex however is formed only in the presence of VraE (Popella et al., 2016). Interestingly, VraH is only important for resistance against gallidermin. Nisin and bacitracin resistance appears to be independent of $\mathrm{VraH}$. This is rather unexpected since gallidermin and nisin share the same structural and mechanistic features, whereas bacitracin is structurally unrelated. Additionally, gallidermin does not form pores in the membranes of most bacteria in contrast to nisin, indicating another mode of action (Popella et al., 2016).

The BraRS TCS has been shown to be specific for nisin and no upregulation occurred when using other antibiotics like vancomycin, fosfomycin, oxacillin, colistin, capreomycin, viomycin, or daptomycin (Hiron et al., 2011).

\section{The NsrFP-RK System from S. agalactiae}

The NsrFP-RK system from $S$. agalactiae is composed of the NBD NsrF (28 kDa), the TMD NsrP $(74 \mathrm{kDa})$, the RR NsrR $(25 \mathrm{kDa})$, and the HK NsrK $(31 \mathrm{kDa})$ (characteristics of the proteins are listed in Table 2; Khosa et al., 2013). Further, the NsrFP-RK system includes an additional serine protease SaNSR, which inactivates nisin by cleaving off the last six amino acids. $\mathrm{SaNSR}$ is anchored in the membrane via a single transmembrane segment (Khosa et al., 2016a). This system confers resistance to multiple lantibiotics such as nisin $\mathrm{A}$, nisin $\mathrm{H}$, and gallidermin as determined with growth inhibition experiments in L. lactis (Khosa et al., 2013; Reiners et al., 2017). Furthermore, it was shown by SYTOX-green assay, that the resistance conferred 
by the $\mathrm{ABC}$ transporter NsrFP is imparted by the inhibiting pore formation in the cell membrane and similar to the CprABC system (see above), it also recognizes specifically the N-terminal part of lantibiotics (Reiners et al., 2017), suggesting a common substrate specificity between these systems. For NsrFP, this was shown by a comprehensive mutational analysis of nisin and comparison of the fold of resistance (Reiners et al., 2017), thereby sensitively quantifying and comparing the growth inhibition studies between lantibiotics and their variants (AlKhatib et al., 2014a,b; Reiners et al., 2017). An advantage of the NsrFP system is their heterologous expression in L. lactis, which allows a mutational analysis, and holds true for the predominant substrate nisin A. Furthermore, the corresponding NsrR (RR) and NsrK (HK) are not present, which allowed the characterization of the NsrFP ABC transporter alone.

For NsrFP, an efflux transport activity has been reported (Reiners et al., 2017). Here, a peptide release assay revealed the transport direction of NsrFP. The efflux of nisin was shown by the amount of nisin present in the supernatant of the cell culture in comparison to a strain harboring an empty plasmid as well as a transport deficient mutant of NsrFP. Furthermore, NsrFP is the BceAB-type transporter that actually suggests an efflux transport direction using a peptide release assay. These results are similar to those previously obtained for the lantibiotic immunity transporters NisFEG and SpaFEG from L. lactis and B. subtilis, respectively, which have been shown to export their corresponding lantibiotics (Stein et al., 2003, 2005).

On the contrary, the TCS NsrRK of the Nsr system has been poorly described so far. Here, NsrR belongs to the large OmpR/PhoB subfamily of response regulators. The structure of the regulator NsrR has been solved by X-ray crystallography and a model in active dimeric DNA-bound state was postulated (Khosa et al., 2016b). This model revealed that the amino acids involved in phosphorylation, dimerization, as well as DNA-binding are conserved on sequence level throughout the family of regulators found in the $B c e A B$ resistance systems identified, so far. This suggests that other BceAB-type response regulators will probably have a similar tertiary structural arrangement.

An extra feature of the NsrFP-RK system is the presence of a membrane-associated serine protease in the operon called $\mathrm{SaNSR}$, which cleaves nisin at its C-terminus and the product nisin $_{1-28}$ has been shown to be 20-100 fold less effective against Gram-positive bacteria membranes (Sun et al., 2009; Khosa et al., 2016a).

The structure of $S a$ NSR was solved at $2.2 \AA$ resolution and displays an N-terminal helical bundle, a protease cap and core domain. Within the latter, the highly conserved TASSAEM region is present. This region contains the active site and lies in a hydrophobic tunnel. Extensive computational modeling of the $S a N S R /$ nisin complex revealed that $S a$ NSR specifically recognizes the C-terminus of nisin, more specifically the last two lanthionine rings of nisin ensuring the exact coordination of the nisin cleavage site at the TASSAEM region (Khosa et al., 2016a). This clearly indicates that in contrast to the efflux mechanism of the ABC transporter NsrFP, SaNSR is highly specific to confer resistance solely against nisin $\mathrm{A}$.

\section{FUTURE DIRECTIONS}

Since (multi-) antibiotic resistant bacteria have rapidly evolved during the last decades, the urgent need for novel compounds is increasing. The secretion of antimicrobial peptides by microorganisms represent a giant pool of novel compounds, which can be used as initial lead structures to develop novel antibiotics.

Here, lantibiotics as small ribosomally-synthesized antimicrobial peptides became relevant and due to genome sequencing the number of identified lantibiotics is rapidly growing. Lantibiotics bind to the essential pyrophosphatesugar moiety of the cell wall precursor lipid II. This is in contrast to well-known glycopeptide antibiotics vancomycin and teicoplanin, which bind to the D-Ala-D-alanyl group of lipid II (Draper et al., 2015). Due to this, it is believed that new resistance mechanisms against lantibiotics are hard to establish for bacteria.

However, inherent resistance against lantibiotics and antimicrobial peptides are already present and are mediated by $\mathrm{ABC}$ transporters, Cpr- and Bce-type transporter, which are present in most human pathogenic bacterial strains. This hampers a wide usage of lantibiotics against severe bacterial infections.

The Cpr ABC transporters resembles the known LanFEG transporter found in lantibiotic producer strains, involved in (auto-)immunity suggesting an evolutionary link. In contrast, the Bce-type $\mathrm{ABC}$ transporters appear to be a novel and unique transporter family, interacting directly with the TCS in the presence of the lantibiotic (Khosa et al., 2013; Dintner et al., 2014). Mechanistically, both families are not very wellunderstood. For the Cpr systems, it has been observed that they are able to expel the lantibiotic from the membrane back into the extracellular media. This would suggest that inhibiting the transporter would allow the lantibiotic to penetrate the membrane again. Therefore, a compound specifically targeting the Cpr transporters would be ideal to use as a lead compound ensuring the potent activity of the lantibiotic itself. To achieve this, more knowledge has to be gained about the exact mechanism of these transporters. Although studies have been performed in vivo, the understanding of binding affinities of the lantibiotic toward the transporter as well as some structural studies will clearly be needed in future. Structurally, the Cpr transporter appears to be a different class of $\mathrm{ABC}$ transporter since none of known $\mathrm{ABC}$ transporter structures seems to be an useful template for molecular modeling approaches using available computer tools, which suggest that structural studies will be needed.

The mechanism of the Bce-type transporters is poorly understood. Recently, for the NsrFP system from S. agalactiae, an export function was reported using a peptide release assay (Reiners et al., 2017). Nevertheless, also a flippase activity of the target molecule lipid II of the ABC transporters would fit to the published studies, since the amount of the lantibiotic in the supernatant would also increase, if the target of the lantibiotic is not present anymore. This hypothesis is also in line with the studies of the $\mathrm{Bce} A \mathrm{~B}$ transporter of $B$. subtilis by Kingston et al. (2014). This flippase activity would also explain why these Bce-type transporters appear to have a large substrate 
spectrum and are able to confer resistance against structurally different lantibiotics as well as some antimicrobial peptides. Here a special focus might be present for the uncharacterized $\mathrm{ECD}_{L}$, the hallmark of BceAB transporters. The function of this $\mathrm{ECD}_{L}$ is unknown, the structure remains elusive so far and further it is not proven that it interacts with the lantibiotic. Therefore, studies revealing function of this $\mathrm{ECD}_{L}$ would likely give a starting point for studies toward the identification of an inhibitor. Remarkable is the complex formation of the BceAB transporter with the TCS. This unusual partnership within the membrane results in a macromolecular complex, which is induced by the presence of the lantibiotic. A pioneering study of the BceAB system from $B$. subtilis suggests that a bacitracin-UPP complex is recognized by $\mathrm{BceAB}$, recognized by the C-terminal part of the TMD BceB up to helix VIII (Kallenberg et al., 2013). Also the complex with the TCS has been shown to be at least stabilized via the TMD. Therefore, studies on the exact function of the $\mathrm{ECD}_{L}$, both biochemically and structurally, will be needed to gain a full understanding of the BceAB system.

Both transporters (BceAB and CprABC) have in common that they are upregulated by a specific TCS induced by the peptide in the medium. Inhibiting the histidine kinase would therefore, be an excellent target for novel drugs, which then in combination with lantibiotics would be a treatment procedure.

Since lantibiotics are active in the low nanomolar range against strains without resistance mechanisms, their potential is clearly demonstrated. If the resistance mechanisms of both

\section{REFERENCES}

Alkhatib, Z., Abts, A., Mavaro, A., Schmitt, L., and Smits, S. H. (2012). Lantibiotics: how do producers become self-protected? J. Biotechnol. 159, 145-154. doi: 10.1016/j.jbiotec.2012.01.032

AlKhatib, Z., Lagedroste, M., Fey, I., Kleinschrodt, D., Abts, A., and Smits, S. H. (2014a). Lantibiotic immunity: inhibition of nisin mediated pore formation by NisI. PLoS ONE 9:e102246. doi: 10.1371/journal.pone.0102246

AlKhatib, Z., Lagedroste, M., Zaschke, J., Wagner, M., Abts, A., Fey, I., et al. (2014b). The C-terminus of nisin is important for the ABC transporter NisFEG to confer immunity in Lactococcus lactis. Microbiologyopen 3, 752-763. doi: $10.1002 / \mathrm{mbo} .205$

Alvarez-Sieiro, P., Montalban-Lopez, M., Mu, D., and Kuipers, O. P. (2016). Bacteriocins of lactic acid bacteria: extending the family. Appl. Microbiol. Biotechnol. 100, 2939-2951. doi: 10.1007/s00253-016-7343-9

Bernard, R., Guiseppi, A., Chippaux, M., Foglino, M., and Denizot, F. (2007). Resistance to bacitracin in Bacillus subtilis: unexpected requirement of the $\mathrm{Bce} A \mathrm{~B} \mathrm{ABC}$ transporter in the control of expression of its own structural genes. J. Bacteriol. 189, 8636-8642. doi: 10.1128/JB.01132-07

Bierbaum, G., and Sahl, H. G. (2009). Lantibiotics: mode of action, biosynthesis and bioengineering. Curr. Pharm. Biotechnol. 10, 2-18. doi: 10.2174/138920109787048616

Brumfitt, W., Salton, M. R., and Hamilton-Miller, J. M. (2002). Nisin, alone and combined with peptidoglycan-modulating antibiotics: activity against methicillin-resistant Staphylococcus aureus and vancomycin-resistant enterococci. J. Antimicrob. Chemother. 50, 731-734. doi: 10.1093/jac/dkf190

Chatterjee, C., Paul, M., Xie, L., and van der Donk, W. A. (2005). Biosynthesis and mode of action of lantibiotics. Chem. Rev. 105, 633-684. doi: 10.1021/cr030105v

Cleveland, J., Montville, T. J., Nes, I. F., and Chikindas, M. L. (2001). Bacteriocins: safe, natural antimicrobials for food preservation. Int. J. Food Microbiol. 71, 1-20. doi: 10.1016/S0168-1605(01)00560-8 transporter families are understood, the first step to inhibit these has been taken, lantibiotics and its variants thereof will be able to fulfill their whole antimicrobial potential.

With novel lantibiotics being identified in new sequenced genomes, the full potential of these antimicrobial peptides has likely not been explored. Probably, for every new lantibiotic discovered a specific resistance system may be present in some human pathogens. Since all lantibiotics target a similar molecule within the target membrane, it is plausible that these resistance mechanisms will be similar to the Cpr and Bce systems, therefore they serve as model systems for lantibiotic resistance.

\section{AUTHOR CONTRIBUTIONS}

RC, JZ-K, and SS wrote the manuscript. RC and SK prepared the figures. RC, JZ-K, SK, and SS finalized the manuscript.

\section{FUNDING}

We gratefully acknowledge funding of the graduate school 2158 (GRK2158) of the Deutsche Forschungsgesellschaft (DFG).

\section{ACKNOWLEDGMENTS}

The authors would like to thank Lutz Schmitt and the Institute of Biochemistry for fruitful discussions and their support. We would like to thank Till Harrar for database searches.
Cotter, P. D., Hill, C., and Ross, R. P. (2005a). Bacterial lantibiotics: strategies to improve therapeutic potential. Curr. Protein Pept. Sci. 6, 61-75. doi: $10.2174 / 1389203053027584$

Cotter, P. D., Hill, C., and Ross, R. P. (2005b). Bacteriocins: developing innate immunity for food. Nat. Rev. Microbiol. 3, 777-788. doi: 10.1038/nrmicro1273

Cotter, P. D., Ross, R. P., and Hill, C. (2012). Bacteriocins-a viable alternative to antibiotics? Nat. Rev. Microbiol. 11, 95-105. doi: 10.1038/nrmicro2937

Delves-Broughton, J., Blackburn, P., Evans, R., and Hugenholtz, J. (1996). Applications of the bacteriocin, nisin. Antonie Van Leeuwenhoek 69, 193-202. doi: 10.1007/BF00399424

Dintner, S., Heermann, R., Fang, C., Jung, K., and Gebhard, S. (2014) A sensory complex consisting of an ATP-binding cassette transporter and a two-component regulatory system controls Bacitracin resistance in Bacillus subtilis. J. Biol. Chem. 289, 27899-27910. doi: 10.1074/jbc.M114. 596221

Dintner, S., Staron, A., Berchtold, E., Petri, T., Mascher, T., and Gebhard, S. (2011). Coevolution of $\mathrm{ABC}$ transporters and two-component regulatory systems as resistance modules against antimicrobial peptides in Firmicutes Bacteria. J. Bacteriol. 193, 3851-3862. doi: 10.1128/JB.05175-11

Draper, L. A., Cotter, P. D., Hill, C., and Ross, R. P. (2015). Lantibiotic resistance. Microbiol. Mol. Biol. Rev. 79, 171-191. doi: 10.1128/MMBR.00051-14

Draper, L. A., Ross, R. P., Hill, C., and Cotter, P. D. (2008). Lantibiotic immunity. Curr. Protein Pept. Sci. 9, 39-49. doi: 10.2174/138920308783565750

Falord, M., Karimova, G., Hiron, A., and Msadek, T. (2012). GraXSR proteins interact with the VraFG $\mathrm{ABC}$ transporter to form a five-component system required for cationic antimicrobial peptide sensing and resistance in Staphylococcus aureus. Antimicrob. Agents Chemother. 56, 1047-1058. doi: 10.1128/AAC.05054-11

Fritz, G., Dintner, S., Treichel, N. S., Radeck, J., Gerland, U., Mascher, T., et al. (2015). A new way of sensing: need-based activation of antibiotic resistance by a flux-sensing mechanism. mBio 6:e00975. doi: 10.1128/mBio.00975-15 
Gebhard, S. (2012). ABC transporters of antimicrobial peptides in Firmicutes bacteria-phylogeny, function and regulation. Mol. Microbiol. 86, 1295-1317. doi: $10.1111 / \mathrm{mmi} .12078$

Gebhard, S., and Mascher, T. (2011). Antimicrobial peptide sensing and detoxification modules: unravelling the regulatory circuitry of Staphylococcus aureus. Mol. Microbiol. 81, 581-587. doi: 10.1111/j.1365-2958.2011.07747.x

Héchard, Y., and Sahl, H.-G. (2002). Mode of action of modified and unmodified bacteriocins from Gram-positive bacteria. Biochimie 84, 545-557. doi: 10.1016/S0300-9084(02)01417-7

Heijenoort, J. V. (1994). Biosynthesis of the bacterial peptidoglycan unit. New Compr. Biochem. 27, 39-54. doi: 10.1016/S0167-7306(08)60406-8

Hiron, A., Falord, M., Valle, J., Débarbouillé, M., and Msadek, T. (2011). Bacitracin and nisin resistance in Staphylococcus aureus: a novel pathway involving the BraS/BraR two-component system (SA2417/SA2418) and both the BraD/BraE and VraD/VraE ABC transporters. Mol. Microbiol. 81, 602-622. doi: 10.1111/j.1365-2958.2011.07735.x

Jung, G. (1991). Lantibiotics-ribosomally sythesized biologically acve polypeptides containing sulfide bridges and $\alpha, \beta$-didehyroamino acids. Angew. Chem. Int. Edn. English 30, 1051-1068. doi: 10.1002/anie.199110513

Kallenberg, F., Dintner, S., Schmitz, R., and Gebhard, S. (2013). Identification of regions important for resistance and signalling within the antimicrobial peptide transporter BceAB of Bacillus subtilis. J. Bacteriol. 195, 3287-3297. doi: 10.1128/JB.00419-13

Kawada-Matsuo, M., Oogai, Y., Zendo, T., Nagao, J., Shibata, Y., Yamashita, Y., et al. (2013a). Involvement of the novel two-component NsrRS and LcrRS systems in distinct resistance pathways against Nisin A and Nukacin ISK-1 in Streptococcus mutans. Appl. Environ. Microbiol. 79, 4751-4755. doi: 10.1128/AEM.00780-13

Kawada-Matsuo, M., Yoshida, Y., Zendo, T., Nagao, J., Oogai, Y., Nakamura, Y., et al. (2013b). Three distinct two-component systems are involved in resistance to the class I bacteriocins, nukacin ISK-1 and nisin A, in Staphylococcus aureus. PLoS ONE 8:e69455. doi: 10.1371/journal.pone.0069455

Khosa, S., Alkhatib, Z., and Smits, S. H. (2013). NSR from Streptococcus agalactiae confers resistance against nisin and is encoded by a conserved nsr operon. Biol. Chem. 394, 1543-1549. doi: 10.1515/hsz-2013-0167

Khosa, S., Frieg, B., Mulnaes, D., Kleinschrodt, D., Hoeppner, A., Gohlke, H., et al. (2016a). Structural basis of lantibiotic recognition by the nisin resistance protein from Streptococcus agalactiae. Sci. Rep. 6:18679. doi: 10.1038/srep18679

Khosa, S., Hoeppner, A., Gohlke, H., Schmitt, L., and Smits, S. H. (2016b). Structure of the response regulator NsrR from Streptococcus agalactiae, which is involved in lantibiotic resistance. PLoS ONE 11:e0149903. doi: 10.1371/journal.pone.0149903

Kingston, A. W., Zhao, H., Cook, G. M., and Helmann, J. D. (2014). Accumulation of heptaprenyl diphosphate sensitizes Bacillus subtilis to bacitracin: implications for the mechanism of resistance mediated by the BceAB transporter. Mol. Microbiol. 93, 37-49. doi: 10.1111/mmi.12637

Kuipers, O. P., Beerthuyzen, M. M., de Ruyter, P. G., Luesink, E. J., and de Vos, W. M. (1995). Autoregulation of nisin biosynthesis in Lactococcus lactis by signal transduction. J. Biol. Chem. 270, 27299-27304. doi: 10.1074/jbc.270.45.27299

Mascher, T. (2006). Intramembrane-sensing histidine kinases: a new family of cell envelope stress sensors in Firmicutes bacteria. FEMS Microbiol. Lett. 264, 133-144. doi: 10.1111/j.1574-6968.2006.00444.x

Mascher, T., Margulis, N. G., Wang, T., Ye, R. W., and Helmann, J. D. (2003). Cell wall stress responses in Bacillus subtilis: the regulatory network of the bacitracin stimulon. Mol. Microbiol. 50, 1591-1604. doi: 10.1046/j.1365-2958.2003.03786.x

McBride, S. M., and Sonenshein, A. L. (2011). The dlt operon confers resistance to cationic antimicrobial peptides in Clostridium difficile. Microbiology 157, 1457-1465. doi: 10.1099/mic.0.045997-0

Meehl, M., Herbert, S., Götz, F., and Cheung, A. (2007). Interaction of the GraRS two-component system with the VraFG ABC transporter to support vancomycin-intermediate resistance in Staphylococcus aureus. Antimicrob. Agents Chemother. 51, 2679-2689. doi: 10.1128/AAC.00209-07

Ohki, R., Giyanto, Tateno, K., Masuyama, W., Moriya, S., Kobayashi, K., et al. (2003). The BceRS two-component regulatory system induces expression of the bacitracin transporter, BceAB, in Bacillus subtilis. Mol. Microbiol. 49, 1135-1144. doi: 10.1046/j.1365-2958.2003.03653.x

Popella, P., Krauss, S., Ebner, P., Nega, M., Deibert, J., and Gotz, F. (2016). VraH Is the third component of the Staphylococcus aureus VraDEH system involved in gallidermin and daptomycin resistance and pathogenicity. Antimicrob. Agents Chemother. 60, 2391-2401. doi: 10.1128/AAC.02865-15

Qiao, M., Ye, S., Koponen, O., Ra, R., Usabiaga, M., Immonen, T., et al. (1996). Regulation of the nisin operons in Lactococcus lactis N8. J. Appl. Bacteriol. 80, 626-634. doi: 10.1111/j.1365-2672.1996.tb03267.x

Reiners, J., Lagedroste, M., Ehlen, K., Leusch, S., Zaschke-Kriesche, J., and Smits, S. H. (2017). The N-terminal region of nisin is important for the BceAB-type $\mathrm{ABC}$ transporter NsrFP from Streptococcus agalactiae $\mathrm{COH} 1$. Front. Microbiol. 8:1643. doi: $10.3389 /$ fmicb.2017.01643

Rietkötter, E., Hoyer, D., and Mascher, T. (2008). Bacitracin sensing in Bacillus subtilis. Mol. Microbiol. 68, 768-785. doi: 10.1111/j.1365-2958.2008.06194.x

Saier, M. H. Jr., Yen, M. R., Noto, K., Tamang, D. G., and Elkan, C. (2009). The transporter classification database: recent advances. Nucleic Acids Res. 37, D274-D278. doi: 10.1093/nar/gkn862

Siegers, K., and Entian, K. (1995). Genes involved in immunity to the lantibiotic nisin produced by Lactococcus lactis 6F3. Appl. Environ. Microbiol. 61, 1082-1089.

Singh, M., and Sareen, D. (2014). Novel LanT associated lantibiotic clusters identified by genome database mining. PLoS ONE 9:e91352. doi: 10.1371/journal.pone. 0091352

Staron, A., Finkeisen, D. E., and Mascher, T. (2011). Peptide antibiotic sensing and detoxification modules of Bacillus subtilis. Antimicrob. Agents Chemother. 55, 515-525. doi: 10.1128/AAC.00352-10

Stein, T., Heinzmann, S., Düsterhus, S., Borchert, S., and Entian, K.-D. (2005). Expression and functional analysis of the subtilin immunity genes spaIFEG in the subtilin-sensitive host Bacillus subtilis MO1099. J. Bacteriol. 187, 822-828. doi: 10.1128/JB.187.3.822-828.2005

Stein, T., Heinzmann, S., Solovieva, I., and Entian, K.-D. (2003). Function of Lactococcus lactis nisin immunity genes nisI and nisFEG after coordinated expression in the surrogate host Bacillus subtilis. J. Biol. Chem. 278, 89-94. doi: $10.1074 /$ jbc.M207237200

Suárez, J. M., Edwards, A. N., and McBride, S. M. (2013). The Clostridium difficile cpr locus is regulated by a noncontiguous two-component system in response to type A and B lantibiotics. J. Bacteriol. 195, 2621-2631. doi: 10.1128/JB.00166-13

Sun, Z., Zhong, J., Liang, X., Liu, J., Chen, X., and Huan, L. (2009). Novel mechanism for nisin resistance via proteolytic degradation of nisin by the nisin resistance protein NSR. Antimicrob. Agents Chemother. 53, 1964-1973. doi: 10.1128/AAC.01382-08

Tagg, J. R., Dajani, A. S., and Wannamaker, L. W. (1976). Bacteriocins of grampositive bacteria. Bacteriol. Rev. 40, 722-756.

Tracanna, V., de Jong, A., Medema, M. H., and Kuipers, O. P. (2017). Mining prokaryotes for antimicrobial compounds: from diversity to function. FEMS Microbiol. Rev. 41, 417-429. doi: 10.1093/femsre/fux014

van Heel, A. J., de Jong, A., Montalban-Lopez, M., Kok, J., and Kuipers, O. P. (2013a). BAGEL3: automated identification of genes encoding bacteriocins and (non-)bactericidal posttranslationally modified peptides. Nucleic Acids Res. 41, W448-W453. doi: 10.1093/nar/gkt391

van Heel, A. J., Mu, D., Montalban-Lopez, M., Hendriks, D., and Kuipers, O. P. (2013b). Designing and producing modified, new-to-nature peptides with antimicrobial activity by use of a combination of various lantibiotic modification enzymes. ACS Synth. Biol. 2, 397-404. doi: 10.1021/sb3001084

Willey, J. M., and van der Donk, W. A. (2007). Lantibiotics: peptides of diverse structure and function. Annu. Rev. Microbiol. 61, 477-501. doi: 10.1146/annurev.micro.61.080706.093501

Conflict of Interest Statement: The authors declare that the research was conducted in the absence of any commercial or financial relationships that could be construed as a potential conflict of interest.

The reviewer CC and handling Editor declared their shared affiliation.

Copyright (๑) 2018 Clemens, Zaschke-Kriesche, Khosa and Smits. This is an openaccess article distributed under the terms of the Creative Commons Attribution License (CC BY). The use, distribution or reproduction in other forums is permitted, provided the original author(s) or licensor are credited and that the original publication in this journal is cited, in accordance with accepted academic practice. No use, distribution or reproduction is permitted which does not comply with these terms. 